\title{
Radial dynamics of electrons in two-section linear accelerator
}

\author{
Aleksandr Nikolaevich Filatov, Vladimir Kuzmich Shilov \\ National Research Nuclear University MEPhI (Moscow Engineering Physics Institute), Russian Federation
}

\begin{tabular}{l} 
Article Info \\
\hline Article history: \\
Received Sep 18, 2017 \\
Revised May 30, 2018 \\
Accepted June 17, 2018 \\
\hline
\end{tabular}

\section{Keywords:}

Electron linear accelerator Biperiodic slowing structure Cell electromagnetic field Buncher, accelerating section Accelerator aperture Radial and phase paths

\begin{abstract}
This article discusses possibility of harness wiring with the use of focusing system of high frequency eigenfields of accelerating resonators in standing wave linear accelerators on the basis of biperiodic slowing systems. The scopes of business activities and specificity of existing engineering processes applied in industry, especially in chemistry and metallurgy, require for special measures on environmental protection. At present electron linear accelerators operating in pulse mode are used for application purposes. Such accelerators can be characterized by sufficient beam power for efficient industrial use and for environmental protection. The results of numerical analysis of electron dynamics in two-section accelerator upon various initial conditions are presented. The obtained results are applied for development of actual accelerator, calculated and experimental data are given. The performed experimental study confirmed possibility of development of standing wave linear accelerator without external magnetic focusing system with output beam diameter of not higher than $8 \mathrm{~mm}$. The results of calculations of beam dynamics are experimentally verified.
\end{abstract}

Copyright (C) 2019 Institute of Advanced Engineering and Science. All rights reserved.

\section{Corresponding Author:}

Aleksandr Nikolaevich Filatov,

Department of Electrical Engineering (8), Institute of General Professional Training,

National Research Nuclear University MEPhI (Moscow Engineering Physics Institute),

Kashirskoye shosse 31, Moscow, 115409 Russian Federation.

Email: a-filatov-00@mail.ru

\section{INTRODUCTION}

In the energy range of 2-50 $\mathrm{MeV}$ the most popular are travelling wave linear accelerators. However, when a portable accelerator is required with the waveguide length of 0.3-0.5 $\mathrm{m}$, electron energy of 4-8 $\mathrm{MeV}$ and low beam current, then standing wave accelerators have certain advantage [1]. Operation modes of electron linear accelerator (linac) are compared in [2] and reasonability of development of standing wave accelerators for small and moderate energies is described.

Analysis of output properties of decelerating radiation upon defect detection [3], radiation therapy [4], elemental activation analysis [5] demonstrates that major portion of radiation problems in these scopes can be solved by linac with the energy of 4-6 $\mathrm{MeV}$ and pulse current of 50-150 $\mathrm{mA}$. In addition, accelerators used for defect detection and medicine should be portable and with small longitudinal and transversal sizes.

Decrease in sizes and weight of radiation assemblies is an urgent task [6]. From the very beginning this task was solved by transition into X-band. At present decrease in longitudinal sizes can be achieved by transition into standing wave mode [7]. In slowing systems of standing wave linacs a biperiodic slowing system (BSS) is used with high specific shunt resistance and accelerating field gradient [8], it is calculated on the basis of equivalent circuit [9].

In BSS the size of aperture for beam transit is extremely low and is about $10 \mathrm{~mm}$ in diameter. Harness wiring through overall accelerator can be executed by means of external focusing fields, which can be created by solenoids. These solenoids can increase significantly transversal sizes of linacs, which is highly undesirable in defect detection and medicine. One of the issues with the use of standing wave linac is harness 
wiring via BSS aperture. Efficiency and straightforwardness of accelerator design depend on successful solution of problem of particle dynamics.

\section{RESEARCH METHOD}

Steady radial motion of particles in standing wave accelerator with high-frequency (HF) focusing is possible only in the phase region where energy gain is not maximum [10]. It is reasonable to implement $\mathrm{HF}$ focusing mode in accelerating structures with high shunt resistance in order to preserve high rate of energy gain by electrons over total accelerator length. Magnetron with maximum pulse power of $1.5 \mathrm{MW}$ was selected as HF energy source for two-section accelerator. Limited magnetron power did not permit to compensate lower energies gained upon the use of HF focusing, thus, it was decided to implement accelerator operation mode with maximum energy gain by electrons.

Taking into consideration all mentioned above, the particle dynamics in two-section accelerator was calculated as follows:

a. at the first stage longitudinal dynamics of particles was calculated, optimum variants were determined with regard to lower energies as well as parameter ranges of the first cell, when the energy gain by particles was maximum;

b. at the second stage, on the basis of the obtained data, the HF fields in contoured resonators were determined [11], and radial dynamics of particles was calculated; parameters of the first cell were varied in the intervals determined at the first stage, the acquired results were analyzed, and the best variant with regard to radial motion was selected;

c. at the third stage, aiming at the best variant with regard to radial motion, the influence of external focusing magnetic field on motion of particles along overall accelerator was studied.

Therefore, in this approach the calculation of particle radial motion was aimed at clarification of sizes of the first cell of accelerating section, intensity of electric field in it and study of influence of external magnetic field on radial properties of beam. As demonstrated by A.E. Novozhilov [12], the parameters of the first cell mainly determine phase, energy, and radial properties of beam at accelerator output. Thus, it is possible to expect that after clarification of parameters of this cell in preset intervals, it will be possible implement harness wiring of a portion of accelerated beam via accelerator without external focusing elements.

\section{RESULTS}

Figure 1 illustrates schematic view of an accelerator comprised of electron injector, two accelerating sections, and external focusing system.

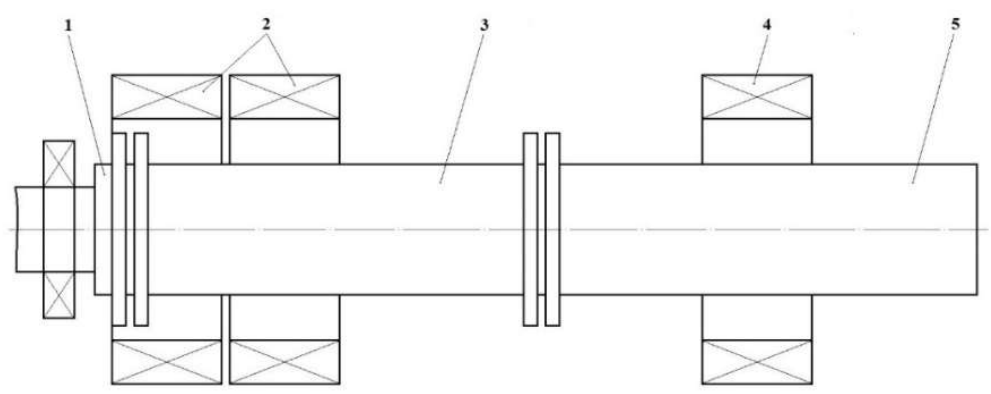

Figure 1. Schematic view of two-section accelerator: 1-injector, 2, 4-focusing coils, 3-the first accelerating section, 5-the second accelerating section

The electron injector at the accelerator output presets a beam with diameter of $1.5 \mathrm{~cm}$, divergences of $1^{\circ}$ and energy of $40 \mathrm{keV}$. These beam parameters will be used as initial for calculation of radial motion of particles. The accelerating sections are based on BSS with end coupling cells and are fed by HF energy via oriented three dB-coupler.

Each accelerating section contains 7 accelerating and 6 end coupling cells. In order to retain electrons in accelerator aperture two coupled coins are installed at input to the first accelerating section and 
single focusing coil at the second section. All coils are made of oxidized aluminum foil and capable to create magnetic field with maximum intensity of $1200 \mathrm{Oe}$.

At the first stage of calculations the optimum accelerator variant was selected with regard to longitudinal particle motion, its parameters are as follows: accelerating field intensity $-200 \mathrm{kV} / \mathrm{cm}$, length$3.2 \mathrm{~cm}$ with the ratio of accelerating gap to cell length equaling to 0.5 . Since the selected accelerating mode of particles is not optimum for radial motion, then at the total accelerator length the beam particles are affected by radial electric field, deflecting from axis, which is directly proportional to accelerating field. With increase in particle energy the influence of this field significantly weakens, but in the accelerator initial portion it is high. The most unfavorable conditions for particle radial motion are in the first cell, into which a beam with the energy of $40 \mathrm{keV}$ is injected. Figure 2 illustrates radial paths of particles in the accelerator variant, selected after the first stage of calculations. Calculations of radial motion at the second stage demonstrated that decrease in electric field intensity in the first cell to $160 \div 170 \mathrm{kV} / \mathrm{cm}$ do not result in significant energy loss at accelerator output.

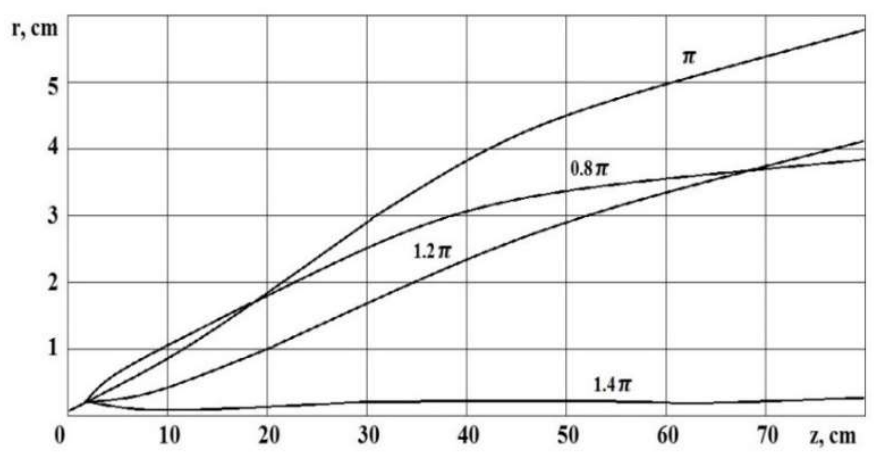

Figure 2. Particle paths in initial accelerator variant, non-optimized with regard to radial motion, for various initial phases of particle entry without external magnetic field

\section{DISCUSSION}

Significant influence on radial motion of particles is exerted by combination of parameters: the length of the first cell and the ratio of accelerating gap of the first cell to its length. This is attributed to the fact that the first cell combines particles into bunches and performs phasing of the bunches with regard to accelerating field in subsequent cells. Optimum parameters of this cell are related with accelerating field intensity in it. As shown by calculations, for $160 \mathrm{kV} / \mathrm{cm}$ the length of the first cell should be selected in the range of $3.5 \div 3.6 \mathrm{~cm}$ at the ratio of accelerating gap to cell length equaling to 0.6 . After analysis of the obtained results at the second calculation stage the parameters of the first cell were adjusted in order to improve radial motion of particles in overall accelerator. Figure 3 illustrates the paths in the adjusted variant.

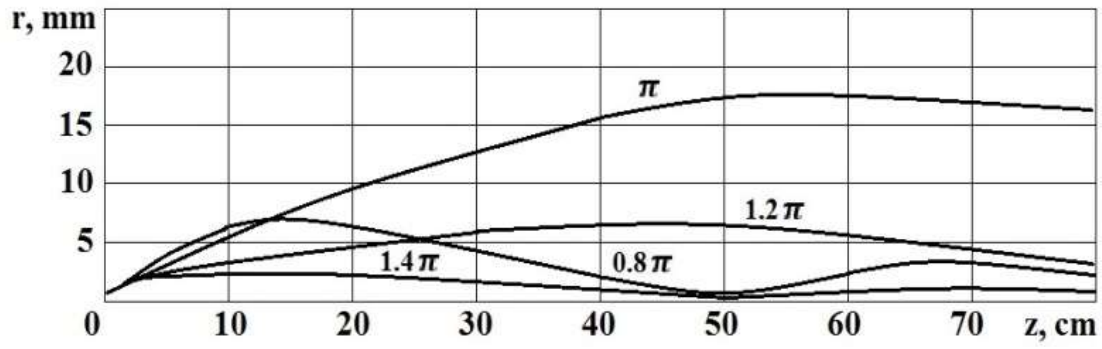

Figure 3. Particle paths in the accelerator variant, optimized with regard to radial motion, for various initial phases without external magnetic field

The improvement of conditions for radial motion in the second case is obvious. Here, without application of external magnetic field, maximum deviation of particles from the axis does not exceed $2 \mathrm{~cm}$, 
and about $50 \%$ of particles captured into acceleration mode only partially escape from the beam channel the radius of which is $0.5 \mathrm{~cm}$. This evidences that using external magnetic field, it is possible to retain all particles in the beam channel aperture. Without external magnetic field it is possible to expect $5 \div 10 \%$ of injected particles at accelerator output.

The third stage of calculations was devoted to analysis of influence of external magnetic field on radial motion, this field formed by two coupled coils at accelerator input and single coil at input. The obtained results evidence that with the $8000 e$ field all particle paths fit into accelerator beam channel aperture. Therefore, the magnetic focusing system is efficient for harness wiring of all particles captured into acceleration mode. Moreover, the calculations demonstrate that the single focusing coil at accelerator output actually does not influence on particle radial motion.

Figure 4 illustrates particle paths for various initial phases at maximum magnetic field intensity of $900 \mathrm{Oe}$ on the axis. It can be seen that the beam diameter does not exceed $6 \mathrm{~mm}$ and all particles captured into acceleration mode reach the accelerator output.

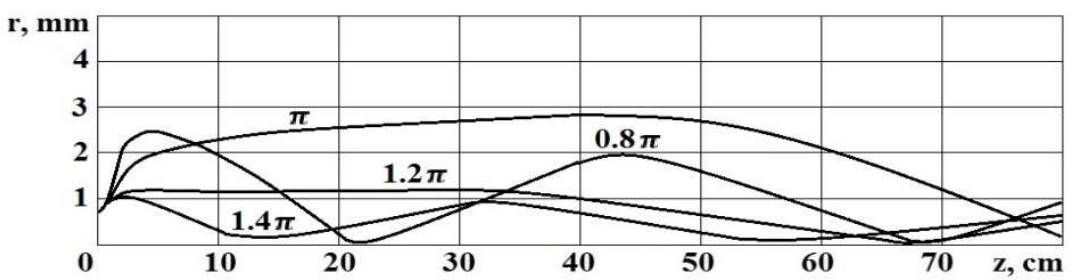

Figure 4. Particle paths in the accelerator variant, optimized with regard to radial motion, for various initial phases with external magnetic field of $900 \mathrm{Oe}$

Therefore, the performed calculations gave the following results:

a. radial dynamics of particles mainly depends on the length of the first cell, the ration of accelerating gap to the length of the first cell, and intensity of accelerating field, these variables in the selected variant are $3.5 \mathrm{~cm}, 0.6$ and $160 \mathrm{kV} / \mathrm{cm}$, respectively;

b. external focusing magnetic system is capable to conduct via accelerator all particles captured in acceleration mode, at magnetic field intensity of $900 \mathrm{Oe}$ the beam diameter at accelerator output is not higher than $2 \mathrm{~mm}$, average kinetic beam energy is $6.7 \mathrm{MeV}$, capturing coefficient is $40 \%$;

c. without external magnetic field $5 \div 10 \%$ of injected particles can be expected at accelerator output.

Since upon implementation of HF focusing mode the beam particles are accelerated not in the maximum $f$ electric field, then it is necessary to estimate unavoidable beam energy loss at accelerator output. With this aim let us use HF beam focusing as a basis of calculation of particle dynamics. Let us determine optimum accelerator variant with regard to radial motion and let us attempt to increase maximally its energy without noticeable impairment of beam radial properties. Let us compare the obtained variant with the previous one, based on maximum possible energy gain by particles.

The accelerator flowchart is the same as in Figure 1, and the sequence of calculations is as follows:

a. calculation of buncher and beam properties, selection of optimum buncher in terms of radial beam formation;

b. calculation of particle dynamics in overall accelerator, analysis of phase motion and energy gain, determination of accelerator parameters influencing energy gain;

c. determination of final variant of accelerator with HF focusing and beam energy properties $[13,14]$.

Let us consider the first two cells of accelerating section as a buncher. Then, it is possible to use the procedure and calculations for two-cell buncher and to assume that the sizes and electric field intensities of the first two cells of the first accelerating section are determined, and the beam properties after the buncher are known. Particle dynamics in overall accelerator are calculated on the basis of the selected accelerator. As assumed, the buncher provide HF focusing mode. It can be obviously seen in Figure 5, where paths of beam particles are illustrated with various initial phases. The buncher forms bunches of $60 \%$ of injected particles, which pass to the accelerator end with average kinetic energy of $3.94 \mathrm{MeV}$. In order to estimate radial properties of beam let us introduce the parameter of average maximum deviation of particle paths from accelerator axis which for this case equals to $0.26 \mathrm{~cm}$. 


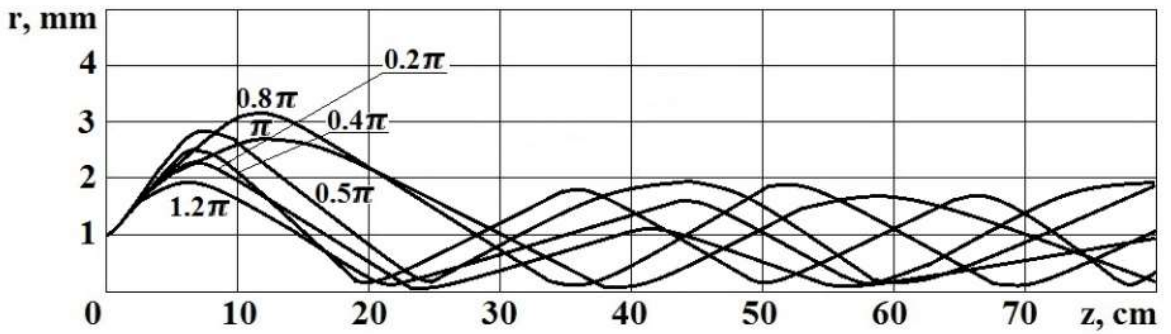

Figure 5. Particle paths in the two-section accelerator variant with HF focusing

\section{CONCLUSION}

The calculations demonstrate that the rate of energy gain by particle to $z=35 \mathrm{~cm}$ is significantly higher than in the remaining portion of accelerator. Indeed, in the first section the energy gain rate is $8 \mathrm{MeV} / \mathrm{m}$, and in the second section it is only $5 \mathrm{MeV} / \mathrm{m}$. A particle passes each subsequent cell in less favorable for acceleration phase than the previous one, that is, the particle gradually slips down from the bump of accelerating field. This slipping can be slightly decreased by variation of length of the second cell. When we decrease the cell length, moderate benefit in energy is obtained, though, the beam radial property sharply deteriorates to $0.5 \mathrm{~cm}$. This evidences the fact that at the accelerator aperture of $1 \mathrm{~cm}$ a portion of beam will obviously fall onto the walls of beam channel.

The worst conditions for phase motion of particle exist in the second section. It is possible to improve these conditions by decreasing the distance between accelerating sections. Optimum results are obtained at the intersection distance of $1.25 \mathrm{~cm}$, thus, this variant can be considered as final, since all particles captured into accelerating mode are held in the accelerator aperture due to HF focusing. The variant of twosection accelerator with HF focusing is characterized by the following output beam parameters: average kinetic energy $-5.7 \mathrm{MeV}$, capturing coefficient $-60 \%$, beam diameter at accelerator output $-8 \mathrm{~mm}$.

Comparison of the obtained variant with the previous one, based on the condition of maximum energy gain by beam with external focusing field, demonstrates that upon implementation of HF focusing mode, the unavoidable loss of energy beam will be $1 \mathrm{MeV}$, however, the capturing coefficient increases by two fold. For the considered accelerator with the length of $75 \mathrm{~cm}$, the beam energy decrease in less than $20 \%$ of maximum value.

\section{ACKNOWLEDGEMENTS} 27.08.2013).

This work was supported by MEPhI Academic Excellence Project (contract No. 02.a03.21.0005,

\section{REFERENCES}

[1] M. Y. Mat Zain, M.T. Ali, A. N. H. Hussin, "High Voltage Durability of Bambusa Vulgaris as a Bio-Composite Material," International Journal of Electrical and Computer Engineering (IJECE), vol 8, pp. 2643-2649, 2018.

[2] S. Hafiz, Zarimin Zaharudin, Sheroz Khan, Nur Shahida Binti Midi, John Tan Teng Hwang, Kushsairy Abdul Kadir, Jawad Ali Shah, "Resonant Configuration Topology Exploration for Inductive Link Power Transfer," Indonesian Journal of Electrical Engineering and Computer Science, vol. 11, pp. 522-530, August 2018.

[3] S.R. Reddy, P.V. Prasad, G.N. Srinivas, "Design of PI and Fuzzy Logic Controllers for Distribution Static Compensator," International Journal of Power Electronics and Drive Systems (IJPEDS), vol. 9, pp. 465-477, 2018.

[4] Ary Syahriar, Nabil Rayhan Syahriar, Jusman Syafii Djamal, "Modelling Optical Waveguide Bends by the Method of Lines," TELKOMNIKA (Telecommunication, Computing, Electronics and Control), vol. 16, pp. 1490-1499, August 2018.

[5] A. E. Novozhilov, et al., "Assessment of Method Errors in Measurement of Acceleration Fields in Accelerating Sections of Charged Particle Accelerators," Life Science Journal, vol. 11, pp. 506-510, 2014.

[6] Motamarri, H.K., Leela Kumari, B, "On-chip Generation of Functional Tests with Reduced Delay and Power," Bulletin of Electrical Engineering and Informatics (BEEI),vol. 6, pp. 36-46, 2017.

[7] Pushan Kumar Dutta, Mircea Bogdan Tătaru, Ovidiu Moldovan, Tiberiu Vesselenyi, "A Case Study of Natural Frequency of the Tram Rail Due to Vibration Using Wavelets, " Indonesian Journal of Electrical Engineering and Informatics (IJEEI), vol 6, pp. 1-11, 2018.

[8] A.E. Novozhilov, et al., "Distribution of Accelerating Voltage in Resonator of Linear Electron-Positron Collider," International Journal of Applied Engineering Research, vol 11, pp. 1596-1602, 2016. 
[9] V.E. Kalyuzhnyi, et al., "Relative Jitter of Accelerating Voltage Amplitude on Resonator's Cells of Linear Electronpositron Collider TESLA-ILC," World Applied Sciences Journal, vol. 27, pp. 1620-1624, 2013.

[10] A.E. Novozhilov, et al., "Problems of Beam Focusing by Electromagnetic Field in Linear Electron Accelerator with Standing Wave, Based on Biperiodic Structure," Modern Applied Science, vol 9, pp. 160-169, 2015.

[11] A.E. Novozhilov, et al., "Calculation of Resonant Frequencies and Electromagnetic Fields in Resonators of Linear Accelerators for Commercial Application, Medicine and Environmental Protection," Research Journal of Pharmaceutical, Biological and Chemical Sciences, 7(2), pp. 897-905, 2016.

[12] A.E. Novozhilov, et al., "Calculation of Bunchers in Linear Electron Accelerators with Standing Wave," ARPN Journal of Engineering and Applied Sciences, vol. 12, pp. 182-187, 2017.

[13] A.E. Novozhilov, et al., "Problems of Measurement of High-Frequency Fields in Linear Electron Accelerators," Global Journal of Pure and Applied Mathematics, vol. 12, pp. 643-655, 2016.

[14] A.N. Filatov, \& V.K. Shilov. "Control of Radio-Frequency Characteristics of Linear Electron Accelerator," Instruments and Experimental Techniques New York, vol 28, pp. 1258-1261, November 1985. 